\title{
Extraction of the Essential Region in Computer-aided Diagnosis of Gastric Atorophy Using Gastric X-ray Images
}

\author{
Koji Abe $^{*}$, Kota Shirakawa ${ }^{\dagger}$, \\ Masahide Minami $\$$, Kenji Yoshikawa $§$
}

\begin{abstract}
To design a computer-aided diagnosis of gastric atrophy, this paper presents a method for extracting the essential region for the diagnosis from gastric X-ray images automatically. Although the automated extraction of gastric area in the X-ray images has a core role as a pre-processing method in the diagnosis, the extraction had been avoided in all the existing systems for diagnosing gastric abnormality because the contour of gastric area is not always clear and other objects often overlap with the contour in the Xray images. Hence, in this paper, an extraction of only the essential region for the diagnosis is investigated. First, two objects of the barium-pool and the spinal column are extracted. Second, with the clue of these objects and difference of density distributions between the gastric area and its outer area, the essential region is extracted by detecting the contour of gastric area. Experimental results for the proposed method using 117 gastric X-ray images have shown that the pro-posed method correctly extracted the essential region from 95 images. This result means most of the existing CAD systems for gastric X-ray images could work well in the case when the proposed method is applied into the systems.
\end{abstract}

Keywords: computer-aided diagnosis, gastric atrophy, medical image processing

\section{Introduction}

In Japan, mass screenings for gastric cancer are conducted for the purpose of its early de-tection and prevention. The Ministry of Health, Labor and Welfare, which is a ministry of Japanese government, recommends the inspection of stomach by endoscopy or X-ray photography making a guideline on cancer screenings [1]. The inspection by $\mathrm{X}$-ray pho-tography is applied for a mass screening and diagnosticians read gastric shape and atrophy [2]. If participants are diagnosed as an abnormal case, the participants have a re-inspection by endoscopy. Thus, the inspection by X-ray photography is conducted as the purpose of discriminating between normal and re-inspection cases. However, the

\footnotetext{
* Faculty of Science and Engineering, Kindai University, Osaka, Japan

$\dagger$ Graduate School of Science and Engineering, Kindai University, Osaka, Japan

\# Graduate School of Medicine, The University of Tokyo, Tokyo, Japan

$\S$ NTT Business Solutions, Osaka, Japan
} 
inspection by X-ray photography has the following problems: 1) the case the diagnosis differs between diag-nosticians is often occurred because the diagnosis is strongly depended on experience of reading the photos, 2) diagnosis by the X-ray photography needs a hard labor because diagnosticians need to read hundreds of photos at a time, and 3) some diagnosticians gradually change diagnosis while they are reading hundreds of the photos at a time. To cope with these problems, a system of computer-aided diagnosis (CAD) which quantitatively evalu-ates gastric abnormality from gastric X-ray images could be effective.

CAD systems for diagnosing the abnormality using the X-ray images have been re-ported [3][4][5][6][7][8]. Although the systems need to extract an essential region for the diagnosis automatically from the images before the diagnosis, the region extraction has been avoided by giving priority to a proposal of discriminating the abnormality. Since the inspection for stomach by X-ray photography is conducted as a mass screening and the number of the images is many, a manual handling should not be contained into the CAD systems. There is a report in which a method for extracting the gastric area from X-ray im-ages using Snake algorithm [9] was presented [10]; however, its application range is very small and the method could work well to only the cases when the contour of the gastric area is appeared clearly. In most of the X-ray images, shades of several 3D objects such as ribs, the spinal column, barium-pools overlap each other on 2D space. In addition, the contour is unclear in most of the X-ray images.

On the other hand, there is another report of a method for extracting the essential region for diagnosing gastric cancer from the images [11]. This method was proposed as a pre-processing of CAD systems for gastric cancer using the images [8]. However, currently, reading points for the X-ray images in the mass screening has been changed and diagnosticians read progression of gastric atrophy in addition to fold patterns of gastric cancer [2]. In reading gastric atrophy, the diagnosticians need to read a wider area than gastric cancer. Therefore, the method brings missing area in applying for extracting the essential region for diagnosing the atrophy. For the reason, this paper proposes a method for extracting the essential region for diagnosing gastric atrophy from gastric $\mathrm{X}$ ray images. The extraction of the region has an effective role of bringing all the existing CAD systems for gastric X-ray images to full-auto systems.

\section{Gastric X-ray Images and the Essential Region for Diagnosing Gastric Atrophy}

In X-ray gastric photography, participants drink barium and radiologists take several X-ray photographs changing direction. Diagnosticians first read the photograph taken from the front shown in Figure 1(a). And, when it is difficult to read the photo due to an unclear case, they read the other photos as the second material. In all the existing CAD systems of diagnosing stomach with X-ray images, the diagnosis is conducted to only the images taken from the front. Hence, in this paper, the proposed method extracts the essential region for the diagnosis from the images as well. Throughout this paper, the size of all the images is $1024 \times 1024$ pixels with 256 gray levels.

The image shown in Figure 1(b) is the copy of Figure 1(a), where a stomach appears in the outer circle and the area $\mathrm{A}$ is the barium pool, the area $\mathrm{B}$, which appears as a band in the vertical direction, is the spinal column, and the area $\mathrm{C}$, which is enclosed by the black line, is the essential region for diagnosing gastric atrophy. In the diagnosis, diagnosticians read folds which mirror the shade of gastric wall and roughness on the wall in the area $\mathrm{C}$. 


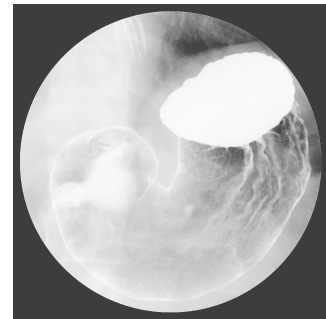

(a) original image

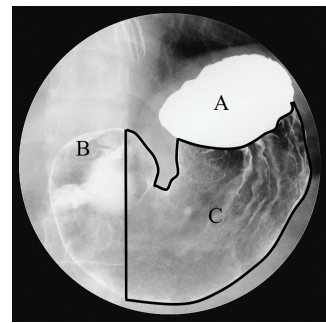

(b) main parts

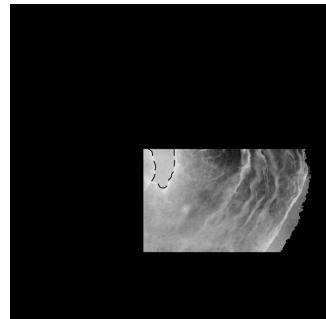

(c) result by the method in [11]

Figure 1:Gastric X-ray image and the essential region for the diagnosis (the area $\mathrm{C}$ in (b)).

On the other hand, Figure 1(c) is the result obtained by applying the existing method for extracting the essential region presented in [11] to the image shown in Figure 1(a). Thus, in [11], since the essential region was defined as the area which is enclosed by the right side line of the spinal column, the horizontal line through the bottom of the barium pool, and the right side contour of gastric area, applying the existing method for diagnosing gastric atrophy could cause lacks of the essential region in many cases. To diagnose the atrophy, diagnosticians read elongation of folds from the bottom of the barium pool to the bottom of the gastric area. And, in Figure 1(c), there is the unnecessary region in the diagnosis, i.e., it is the region enclosed by the broken line in Figure 1(c) and it becomes a noise in analyzing abnormality of the atrophy because its gray levels are similar to folds and edges in the X-ray images as shown in Figure 1. Therefore, it is necessary to propose another way for extracting the essential region which keeps folds appeared in gastric area and does not include the unnecessary area.

\section{Proposed Method}

\subsection{Extraction of the Barium Pool}

Since barium is used for clearly showing figure of folds on the gastric wall as shown in Figure 1(a), the brightness of the barium is significantly higher than other parts in the Xray images. Pixel values of the barium pool are certainly between 150 and 255, and the contour of the barium pool appear clearly. Hence, to catch the barium pool, the contour of the barium pool is obtained as follows.

First, if the pixel value is smaller than 150 , it is converted into 0 (i.e., this pixel color becomes black.). Second, the smoothing processes by the moving average with the $3 \times 3$ mask shown in Figure 2 and edge enhancement by Kirsh filtering [12] are applied to the image. For example, the original image shown in Figure 3(a) is converted into Figure 3(b). Third, the image is binarized into the black and white image by linear discriminant analysis, and the thinning is applied to the image. Figure 3(c) shows the image converted by the thinning for Figure 3(b). Since the barium pool is basically located at the upper right of the original image, the maximum value of pixel values is obtained in the quarter area at the upper right side of the image when the image is separated into $2 \times 2$ squares. Forth, in the original image, all the pixels which have pixel values between the maximum value and the value which is smaller than the maximum value by 3 are extracted; besides the centroid point of the extracted area is obtained as the centroid point of the barium pool. Figure 3(d) shows the pixel extraction for Figure 3(a) and the centroid point. Fifth, radiating lines from the centroid point, the intersections between the lines and white pixels in the image 


\begin{tabular}{|c|c|c|}
\hline$\frac{1}{10}$ & $\frac{1}{10}$ & $\frac{1}{10}$ \\
\hline$\frac{1}{10}$ & $\frac{2}{10}$ & $\frac{1}{10}$ \\
\hline$\frac{1}{10}$ & $\frac{1}{10}$ & $\frac{1}{10}$ \\
\hline
\end{tabular}

Figure 2: The mask used for the smoothing.

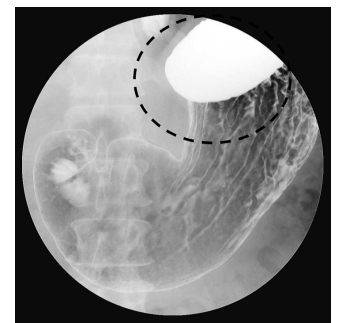

(a) original image

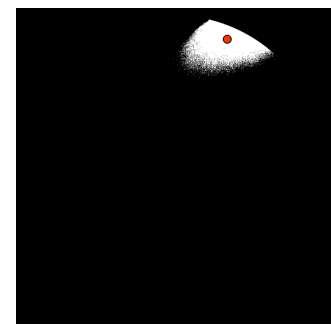

(d) the centroid point

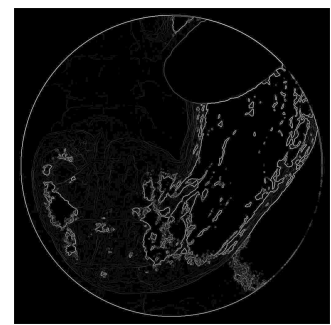

(b) after the filterings

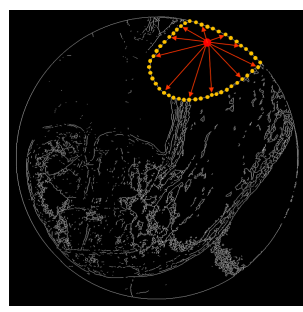

(e) the inersections

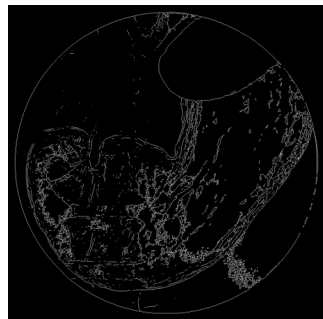

(c) after the thinning

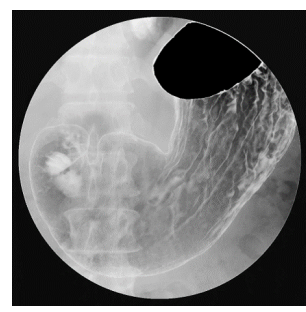

(f) output

Figure 3: Procedure of the extraction of the barium pool. The barium pool is the white area enclosed by the broken line in (a).

obtained by the thinning are obtained. The lines radiate from the point at intervals of the angle of 7.5 degrees, i.e., the number of the extracted intersections becomes 48 points. Figure 3(e) shows the intersections extracted from Figure 3(c) and Figure 3(d). Sixth, when a couple of the adjacent intersections is not connected by white pixels, the couple is connected by a white line. In the area enclosed by the white pixels, if the mean value of pixel values is smaller than 200, or the area size is smaller than 30,000 and the area dose not attach to the outer circle, it is regarded there is no barium pool in the image. Throughout this procedure, the barium pool appeared in Figure 3(a) is finally extracted as shown in Figure 3(f), where the mean value of pixel values and the area size are 248 and 66,126 in the extracted area, respectively.

\subsection{Extraction of the Spinal Column}

The spinal column basically appears like a vertical bar in the images as shown in Figure 1(a). However, since it has a slight slope depending on images, the original image is rotated to become a vertical spinal column as follows. Separating the image into 16 equal parts in the vertical direction, the mean value of pixel values is calculated in each part; besides the maximum value of the 16 mean values is found. Then, all the parts 


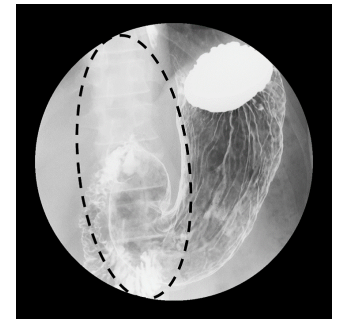

(a) original image

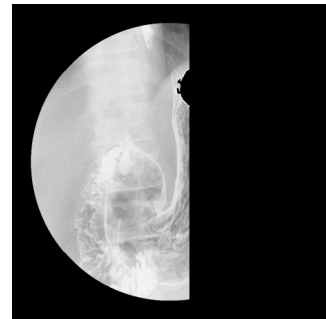

(b) $B(x, y)$

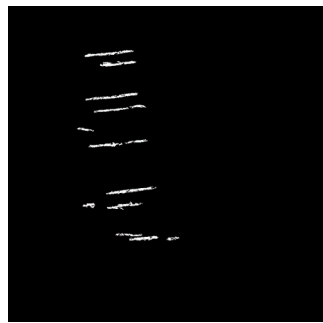

(c) $I(x, y)$

Figure 4: $B(x, y)$ and $I(x, y)$. The spinal column is the longitudinal bar enclosed by the broken line in (a).

which have the mean value between the maximum value and the value which is smaller than the maximum value by 50 are extracted. The image represented by the extracted parts is defined as image $B(x, y)$, and the following procedure is conducted to $B(x, y)$. For example, $B(x, y)$ for the original image as shown Figure 4(a) is obtained as Figure 4(b).

1. The line convergence index $d(x, y)$ and the angle $\theta(x, y)$ are obtained by the line convergence index filtering [13], where $-1<d(x, y)<1$ and $-90<\theta(x, y)<90$.

2. If the pixel $(x, y)$ is satisfied with $d(x, y)>0.8$ and $-12<\theta(x, y)<12$, its pixel color is converted into white. Otherwise, the color is converted into black. The converted image is defined as image $I(x, y)$.

3. if area size of the white region in $I(x, y)$ is smaller than 200 , the white region is removed from $I(x, y)$.

4. The mode value $\theta$ obtained from $\theta(x, y)$ at all the white pixels in $I(x, y)$ is regarded as the slope.

5. $B(x, y)$ and $I(x, y)$ are rotated $-\theta$ degrees with the clockwise direction as positive.

$I(x, y)$ for the $B(x, y)$ shown in Figure 4(b) is obtained as Figure 4(c).

Next, the scanning window $(1024 \times 300(\mathrm{H} / \mathrm{W}))$ is prepared at left side in each of $B(x, y)$ and $I(x, y)$. Scanning the window to the right side in the horizontal direction, the number $n_{x}$ of white pixels and the variance $v_{x}$ of pixel values in the window are obtained from $I(x, y)$ and $B(x, y)$ at every location, respectively. And, the location of the window where $n_{x} / v_{x}$ has the maximum value is found. After then, expanding width of the window by 50 pixels to the left and right at the location, this region $(1024 \times 400(\mathrm{H} / \mathrm{W}))$ is defined as the candidate of the spinal column. Next, another scanning window $(1024 \times 300(\mathrm{H} / \mathrm{W}))$ is newly prepared at the left side of the candidate; besides separating the window into equal three areas in the vertical direction, the three areas are defined as L, M, and R, respectively. Figure 5(a) shows the candidate of the spinal column obtained from Figure 4(b) and Figure 4(c), where the window, L, M, and R are located. The window scans the candidate to the right horizontally. In the window, regarding $x$-coordinate and the mean value of pixel values in each column as $\mathrm{x}$ and $\mathrm{y}$ axes respectively, the approximate line is obtained in each of L, M, and R. Scanning the window, first, all the locations where the mean value of pixel values in $\mathrm{M}$ is larger than 150 are found. Next, among the locations, the location where difference between slopes of the approximate line in $\mathrm{L}$ and $\mathrm{R}$ is the largest is found. The window at the location is extracted as the spinal column. Figure 5(b) shows the spinal column extracted from Figure 5(a). After that, the image is rotated $\theta$ degrees. 


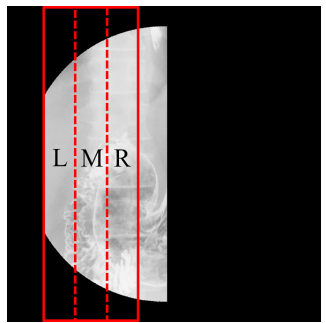

(a) the window and LMR

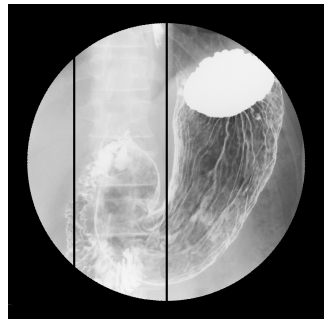

(b) the spinal column extracted from (a)

Figure 5: Extraction of the spinal column.

\subsection{Extraction of the Right Side Contour of Gastric Area}

Removing the left area from the right side line of the extracted spinal column and all the pixels at the upper locations of the extracted barium pool in the original image, image $R(x, y)$ is prepared as shown in Figure 6(a). The line convergence index filtering [13] and high-pass filtering $(7 \times 7)$ [14] are applied to $R(x, y)$, and the line convergence index $d_{R}(x, y)$ and $H p f_{R}(x, y)$ which was converted by the high-pass filtering are obtained, besides a $2 \mathrm{D}$ space which has two axes of $d_{R}(x, y)$ and $H p f_{R}(x, y)$ is obtained. Every pixel of $R(x, y)$ is placed in the space and the pixel is discriminated whether it could be a part of a strong edge or not by $k$-means classification [15]. With the discriminated pixels as white and the other pixels as black, a black and white image is obtained. If the perimeter of the region is smaller than 100 in the image, the region is removed from the image. After that, the image is defined as $E(x, y)$ as shown in Figure 6(b). Then, putting 30 points at even intervals on the boundary of the quadrant in the area at the lower right side in separating the original image into $2 \times 2$ as shown in Figure 6(c), the points are set in $E(x, y)$ as well. Next, every point moves to the central point as shown in Figure 6(d), and when the point reaches the first white pixel in $E(x, y)$, the following procedure is applied to the point.

1. A window of $61 \times 61$ is set in $E(x, y)$. The point is the central point of the window.

2. If the white region which includes the point connects the boundary of the window and separates the window into two areas, the region is regarded as a candidate of the gastric contour.

3. The separated area which includes the upper left end of the window is defined as $A$, and another area is defined as $B$. When the candidate region covers the gastric contour, variance of pixel values in $A$ should be larger than $B$ because many folds appear in the gastric area. Hence, overlapping the white region and its window on $R(x, y)$ and if the variance in $A$ is smaller than $B$, the point is removed from $E(x, y)$. Otherwise, the point is retained in $E(x, y)$. After then, the overlapped white region and its window are removed from $R(x, y)$.

After that, all the white regions which have the points retained in $E(x, y)$ are extracted from $E(x, y)$. The created image is defined as $E_{2}(x, y)$ as shown in Figure 6(e). Next, radiating straight lines from the central point of $E_{2}(x, y)$, only the white regions that the lines first reach are obtained from $E_{2}(x, y)$ as $E_{3}(x, y)$ as shown in Figure 6(f). The lines radiate from the central point at even intervals of the angle of 3 degrees, and range of the radiation is 90 degrees in the lower right area in separating the image into $2 \times 2$ equal squares. Then, the upper column and the left line from every pixel of the obtained regions are converted into white color as shown in Figure 6(g). Besides, if the white regions attach the outer circle in 


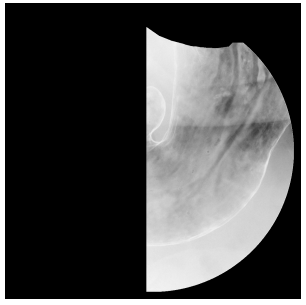

(a) $R(x, y)$

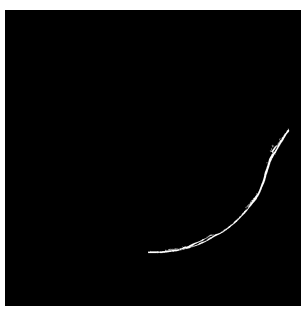

(e) $E_{2}(x, y)$

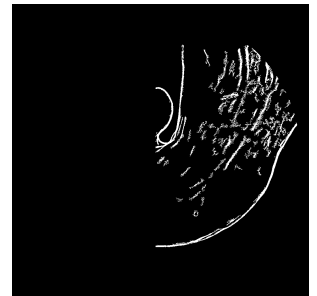

(b) $E(x, y)$

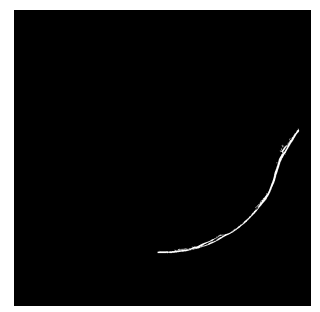

(f) $E_{3}(x, y)$

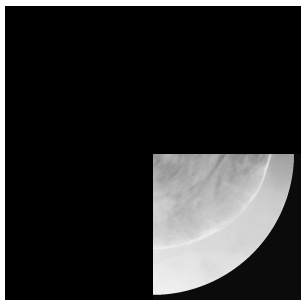

(c) the $1 / 4$ area

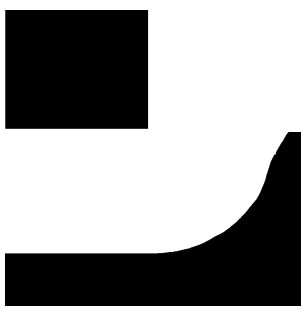

(g) the conversion

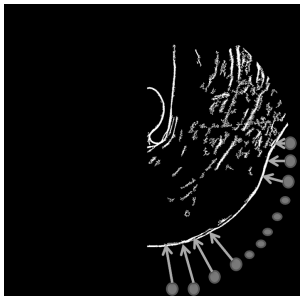

(d) the points

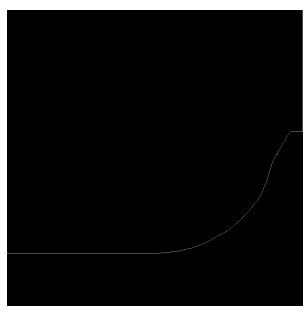

(h) the contour

Figure 6: A flow of the image conversion in the extraction of the contour.

the X-ray image, a horizontal line is drawn from every pixel of the white regions to the right end of the image and the upper area of the line is converted into white color, too. Finally, as shown in Figure 6(h), the boundary between black and white pixels at the right side in the image is extracted as the right side contour of gastric area.

The essential region is obtained by enclosing the barium pool, the right sideline of the spinal column, and the right side contour of gastric area.

\subsection{Noise Removal}

Even the essential region extracted by the proposed method shown above includes the noise shown in 2. Here, it is removed as follows. First, all the pixels between $E(x, 623)$ and $E(x, 1023)$, and between $E(680, y)$ and $E(1023, y)$ are removed. Second, scanning points are set at even intervals of 10 pixels in $E(0, y)$, and each of the points moves to the right direction horizontally until it reaches the first white pixel. Then, to each of the points, the processing 1 and 2 shown in 3.3 is conducted besides the areas $A$ and $B$ are determined as well as the step 3 in 3.3 . Here, on the contrary to the content of the step 3 in 3.3 , if variance of pixel values in $B$ is smaller than $A$, and the mean value of pixels in $A$ is smaller than 170, the point is removed from $E(x, y)$; otherwise, the point is retained in $E(x, y)$. After that, as well as 3.3 , the boundary between the noise and the gastric area is determined as the inner contour of the gastric area and the noise is removed by removing the left side and the upper areas of the contour.

\section{Experimental Results}

The proposed method of extracting the essential region was applied to 117 gastric X-ray images (12 normal and 105 abnormal cases). To prepare correct answers in this experiment, all the cases were diagnosed by an expert diagnostician. And, performance of the extractions (i.e., extraction success or failure) was evaluated by the diagnostician's eyes. Table 1 shows experimental results for the extractions. As shown 
Table 1: Experimental Results

\begin{tabular}{|l|r|r|r|}
\hline & \multicolumn{1}{|l|}{ barium pool } & \multicolumn{1}{l|}{ spinal column } & the essential region \\
\hline Extraction success & 111 cases $(94.9 \%)$ & 111 cases $(94.9 \%)$ & 95 cases $(81.1 \%)$ \\
\hline Extraction failure & 6 cases & 6 cases & 22 cases \\
\hline
\end{tabular}

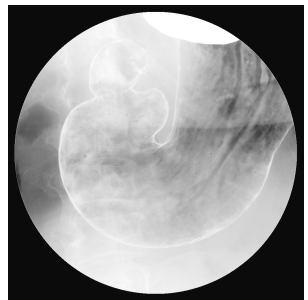

(a) original image

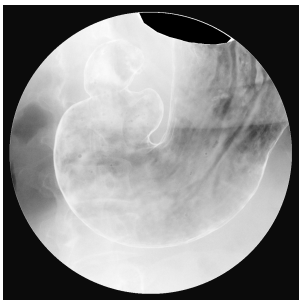

(b) barium pool

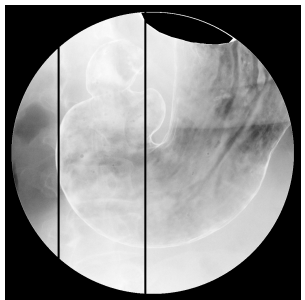

(c) spinal column

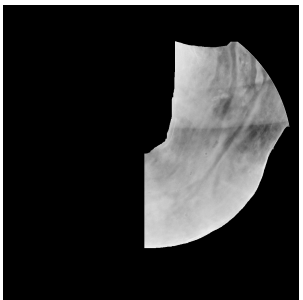

(d) essential region

Figure 7: A sample of the extraction success.

in Table 1, the success ratio of the extraction for the essential region was $81.1 \%$. Figure 7 shows a sample of extraction success for the essential region.

The extraction of the barium pool was evaluated by a criterion of extraction success that pixels of $90 \%$ at least in the extracted area are included in the correct barium pool obtained by hand. In the extraction of the barium pool, the failure 6 cases can be broadly divided into two kinds. The one is the case when the proposed method could not well catch the contour of the barium pool because the contour was broken off due to the edge enhancement. Figure 8(a) shows a sample of this failure cases. To cope with this, it is necessary to include some conditions on the distance from the centroid point to the contour in catching the contour. The other one is the case when the barium pool did not appear and the proposed method extracted another region. Figure 8(b) shows a sample of this failure cases, where the mean value of pixel values was 217 and the area size was 46,967. To cope with this, it is necessary to revise the conditions of the barium pool introducing other characteristics.

The extraction of the spinal column was evaluated by a criterion of extraction success that the extracted area includes the right side contour of the correct spinal column. Figure 9 shows a sample of the failure 6 cases. As shown in Figure 9, the proposed method extracts a region as the spinal column even if the spinal column does not appear. To cope with this, it is necessary to discriminate whether the extracted region correctly includes the spinal column introducing other characteristics.

The extraction of the essential region was evaluated by a criterion of extraction success that the extracted region extracts only the gastric area and it is sufficiently possible to diagnose gastric atrophy. Among the 22 failure cases, 9 cases were the extraction failure due to either of the extraction failures for the barium pool and the spinal column. Except them, the failure cases can be divided into two kinds. The one is the case when the points could not catch the gastric contour correctly because a barium region which appears in the outside of the gastric contour became a noise as shown in Figure 10(a). The other one is the case when the proposed method could not remove the noise as shown in Figure 10(b), i.e., the processing shown in 3.4 was not work well in the cases. To cope with this, it is necessary to revise the conditions for catching the contour. 

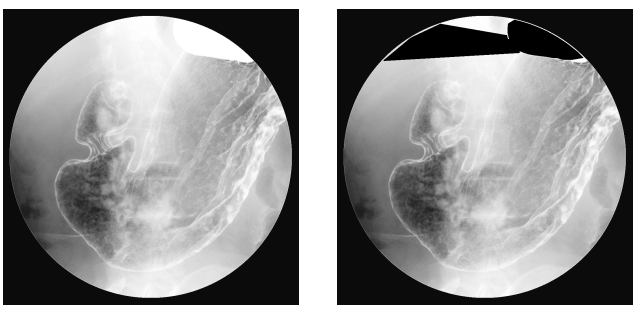

(a) failure case 1
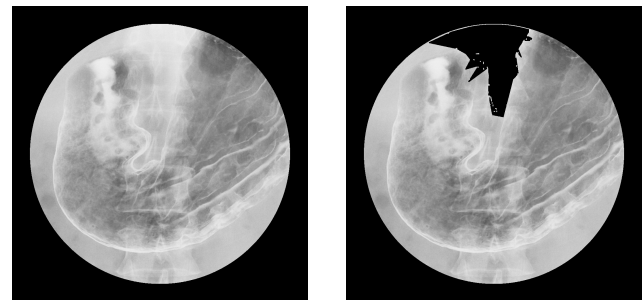

(b) failure case 2

Figure 8: Sample of the extraction failures for the barium pool.

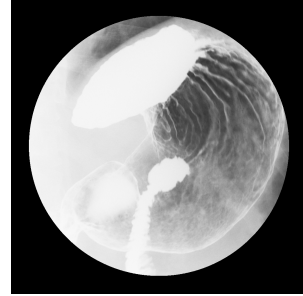

(a) original image

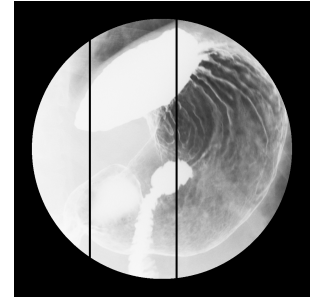

(b) result

Figure 9: A failure case for the spinal column.

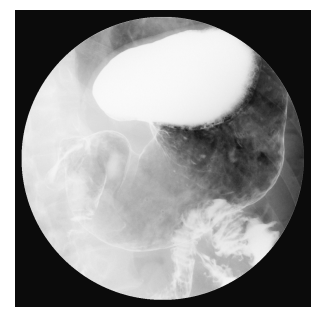

(a) failure case 1
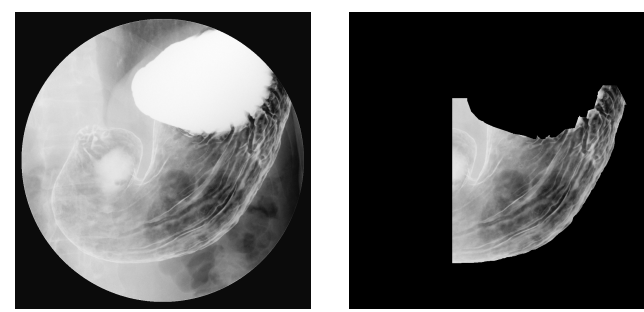

(b) failure case 2

Figure 10: Sample of the extraction failures for the essential region.

\section{Conclusion}

This paper presented a method for extracting the essential region for diagnosing gastric atrophy in X-ray images. In the proposed method, after the barium pool, the spinal column, and a part of the gastric contour were extracted, the essential region was determined by enclosing them and conducting a noise removal. Experimental results for the proposed method using 117 gastric X-ray images showed that the proposed method could extract the region correctly in most cases. As future works, it is necessary to improve the conditions in extracting the barium pools, propose a method for recognize whether there is the spinal column and a method for removing noises located in the outside of the gastric contour.

\section{Acknowledgments}

This work was partially supported by JSPS KAKENHI Grant No.16K00258.

\section{References}

[1] Ministry of Health Labour and Welfare in Japan, Guidelines for implementation of emphasis on cancer prevention and health checkup for cancer; http://www.mhlw.go.jp/file/06-Seisakujouhou-10900000-

Kenkoukyoku/0000111662.pdf (in Japanese). (accessed on 2018/11/13) 
[2] Quality Control Committee for Gastric Cancer Screening in the Japanese Society of Gastrointestinal Cancer Screening, Atras for Classifying Diagnoses in Gastric X-ray Photography, Nankodo, 2017. (in Japanese)

[3] Y. Kita, "Elastic-model driven analysis of several views of a deformable cylindrical object," IEEE Trans. Pattern Anal. Mach. Intel., vol.18, no.12, pp.1150-1162, 1996.

[4] Y. Mekada, J. Hasegawa, J. Toriwaki, S. Nawano, and K. Miyagawa, "Automated extraction of cancer lesions from double contrast X-ray images of stomach," Proc. 1st International Workshop on Computer Aided Diagnosis, pp.407-412, 1998.

[5] J. Hasegawa, T. Tsutsui, and J. Toriwaki, "Automated Extraction of Cancer Lesions with Convergent Fold Patterns in Double Contrast X-ray Images of the Stomach," Systems and Computers in Japan, vol.22, no.7, pp.51-62, 1991.

[6] J. Hasegawa and J. Toriwaki, "A new filter for feature extraction of line pattern texture with application to cancer detection," Proc. 11th IAPR Int. Conf. on Pattern Recognition, pp.352-355, 1992.

[7] Y. Yoshinaga, H. Kobatake, and S. Fukushima, "The detection and feature extraction method of curvilinear convex regions with weak contrast using a gradient distribution method," Proc. IEEE ICIP 99, pp.715-719, 1999.

[8] K. Abe, H. Nakagawa, M. Minami, and H. Tian, "Features for discriminating normal cases in mass screening for gastric cancer with double contrast X-ray images of stomach," Journal of Biomedical Engineering and Medical Imaging, vol.1, issue 6, pp.2232, Dec. 2014.

[9] M. Kass, A. Witkin, and D. Terzopoulos, "Snakes: Active contour models," International J. Computer Vision, vol.1, no.3, pp.321-331, 1988.

[10] S. Fukushima, H. Uwai, and K. Yoshimoto, "Optimization-Based Recognition the Gastric Region from a Double-Contrast Radiogram,” IEICE Trans. (Japanese Edition), vol.J83-D-II, no.1, pp.154-164, Jan. 2000.

[11] K. Abe, H. Nakagawa, M. Minami, and H. Tian, "Extraction of essential region in gastric area for diagnosing gastric cancer using double contrast X-ray images," Proc. of the 11th Australasian Data Mining Conference, pp.9-16, Nov. 2013.

[12] J. R. Parker, Algorithms for Image Processing and Computer Vision, Wiley Publishing, 2010.

[13] Y. Yoshinaga and H. Kobatake, "The line detection method with robustness against contrast and width variation applied in gradient vector field," Systems and Computers in Japan, vol.31, no.3, pp.49-58, 2000.

[14] R. A. Schowengerdt, Techniques for Image Processing and Classification in Remote Sensing, Academic Press, 1983.

[15] M. J. Canty, Image Analysis, Classification and Change Detection in Remote Sensing, CRC Press, 2014. 